\title{
Design, manufacturing, and performance analysis of mid-infrared achromatic half-wave plates with diamond subwavelength gratings
}

\author{
Christian Delacroix, ${ }^{1, *}$ Pontus Forsberg, ${ }^{2}$ Mikael Karlsson, ${ }^{2}$ Dimitri Mawet, ${ }^{3}$ \\ Olivier Absil, ${ }^{4}$ Charles Hanot, ${ }^{4}$ Jean Surdej, ${ }^{4}$ and Serge Habraken ${ }^{1}$ \\ ${ }^{1}$ Hololab, Université de Liège, Allée du 6 Août 17 B5a, B-4000 Liège, Belgium \\ ${ }^{2}$ Ångström Laboratory, Uppsala University, Lägerhyddsvägen 1, SE-751 21 Uppsala, Sweden \\ ${ }^{3}$ European Southern Observatory (ESO), Alonso de Córdova 3107, Vitacura, 7630355 Santiago, Chile \\ ${ }^{4}$ IAGL, Université de Liège, Allée du 6 Août 17 B5c, B-4000 Liège, Belgium \\ ${ }^{*}$ Corresponding author: delacroix@ astro.ulg.ac.be
}

Received 3 May 2012; revised 10 July 2012; accepted 10 July 2012;

posted 20 July 2012 (Doc. ID 167832); published 16 August 2012

\begin{abstract}
In this paper, we present a solution for creating robust monolithic achromatic half-wave plates (HWPs) for the infrared, based on the form birefringence of subwavelength gratings (SWGs) made out of diamond. We use the rigorous coupled wave analysis to design the gratings. Our analysis shows that diamond, besides its outstanding physical and mechanical properties, is a suitable substrate to manufacture mid-infrared HWPs, thanks to its high refractive index, which allows etching SWGs with lower aspect ratio. Based on our optimized design, we manufactured a diamond HWP for the 11-13.2 $\mu \mathrm{m}$ region, with an estimated mean retardance $\sim 3.143 \pm 0.061 \mathrm{rad}\left(180.08 \pm 3.51^{\circ}\right)$. In addition, an antireflective grating was etched on the backside of the wave plate, allowing a total transmittance between $89 \%$ and $95 \%$ over the band. (C) 2012 Optical Society of America
\end{abstract}

OCIS codes: $\quad 050.1950,050.5080,050.6624$.

\section{Introduction}

Wave plates are essential tools for modulating the polarization of an incoming light beam. They are commonly used in many applications as retarders or phase shifters. In astrophysics, when it comes to observing a very high-contrast scene, one must be able to cancel the bright source, or at least drastically reduce its intensity. This attenuation can be achieved by combining two portions of the incident light beam, one of which having to undergo a half wavelength (or $\pi$ ) phase shift. Therefore, a half-wave plate (HWP) is by definition the ideal tool. The retardance of a zero-order wave plate varies hyperbolically with the

$1559-128 \mathrm{X} / 12 / 245897-06 \$ 15.00 / 0$

(C) 2012 Optical Society of America wavelength, which limits the operation of single wave plates to monochromatic light. However, a large spectral bandwidth is needed in many cases, especially in astrophysics, both to increase the signal-to-noise ratio and to allow spectrophotometry.

Different varieties of achromatic wave plates exist. Achromatic prism retarders [1], for instance, operate in total internal reflection. They are voluminous and not suitable for applications operating in transmission. Another way of achieving broadband performance is stacking several crystal wave plates together [2] and orienting their birefringent axes using a Pancharatnam method [3]. A thick combination of multiorder wave plates is needed, because of the weak natural birefringence of crystals, which results in an increased absorption of IR radiation. Achromatic retarders can also be produced by using 
liquid crystals (LCs) [4], liquid crystal polymers (LCPs) [5], or photonic crystals (PhCs) [6, 7] as the birefringent materials. A weakness of these liquid or photonic crystal retarders is their bandwidth: they do not transmit at wavelengths beyond the nearinfrared (H-band centered at $\sim 1.65 \mu \mathrm{m}, \mathrm{K}$-band $\sim 2.2 \mu \mathrm{m}$ ) [8]. Meanwhile, the demand for instruments in the mid-infrared (L-band $\sim 3.8 \mu \mathrm{m}, \mathrm{M}$-band $\sim 4.8 \mu \mathrm{m}$, N-band $\sim 10.5 \mu \mathrm{m}$ ) is increasing in many domains of astrophysics. Therefore, we are pursuing a different technological route to synthesize the $\pi$ phase shift. We use the dispersion of form birefringence of subwavelength gratings (SWGs) $[9,10]$, which are particularly adapted to longer wavelengths.

In this paper, we present the results of our work on achromatic wave plates. After a brief introduction to SWGs (Section 2), we demonstrate in Section 3 that diamond is a good choice of material for mid-IR SWGs. In Section 4, we attempt to optimize a design for a diamond HWP with respect to manufacturability and performance. In Section 5 , we briefly describe the fabrication of a diamon $\bar{d}$ achromatic HWP dedicated to the mid-IR $(11-13.2 \mu \mathrm{m})$. We calculate its theoretical efficiency with computer simulations based on the rigorous coupled wave analysis (RCWA). Finally, we conclude with the perspectives for present and future instruments.

\section{Achromatic Half-wave Plates (HWPs)}

\section{A. Subwavelength gratings (SWGs)}

Many new optical devices have been made with SWGs, such as high-efficiency diffraction gratings, polarization-selective gratings, wave plates, and monolithic antireflective structures [11]. SWGs are micro-optical structures with a period $\Lambda$ smaller than $\lambda / n, \lambda$ being the observed wavelength of the incident light and $n$ the refractive index of the grating substrate. Such structures do not diffract light as a classical spectroscopic grating does. Instead, only zeroth transmitted and reflected orders are allowed to propagate outside the grating, and the incident wavefront is not affected by further aberrations. The condition for having an SWG is defined by the grating equation, which determines whether a diffraction order propagates or not through the grating,

$$
\frac{\Lambda}{\lambda} \leq \frac{1}{n_{I} \sin \theta+\max \left(n_{\mathrm{I}}, n_{\mathrm{II}}\right)},
$$

where $\theta$ is the incidence angle and $n_{\mathrm{I}}$ and $n_{\mathrm{II}}$ are the refractive indices of the incident (superstrate) and transmitting (substrate) media, respectively.

One can employ these SWGs to synthesize artificial birefringent achromatic wave plates [9]. A birefringent medium, such as a grating (see Fig. 1), has two different refractive indices, $n_{\mathrm{TE}}$ and $n_{\mathrm{TM}}$, with regard to the polarization states TE (transverse electric, parallel to the grating grooves) and TM (transverse magnetic, orthogonal to the grating grooves). The phase retardation $\Delta \Phi$ introduced by a birefrin-

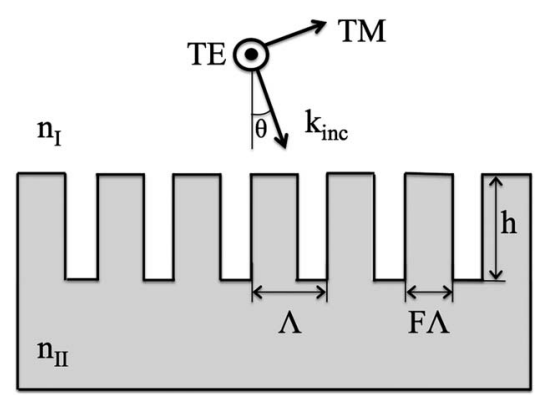

Fig. 1. Schematic diagram of a SWG. The incident light beam vector $k_{\text {inc }}$ is perpendicular to the grating lines. The filling factor $F$ is such that $F \Lambda$ corresponds to the width of the grating walls.

gent SWG between the two polarization components is dependent on the wavelength, and is given by

$$
\Delta \Phi_{\mathrm{TE}-\mathrm{TM}}(\lambda)=\left(\frac{2 \pi}{\lambda}\right) \Delta n_{\mathrm{form}}(\lambda),
$$

where

$$
\Delta n_{\text {form }}(\lambda)=n_{\mathrm{TE}}(\lambda)-n_{\mathrm{TM}}(\lambda) .
$$

$h$ is the optical path through the birefringent medium. In order to produce an achromatic wave plate, the product of the two factors in $\lambda$ on the right-hand side of Eq. (2) needs to be a constant over a wavelength range as large as possible. By varying the grating parameters (geometry, material, incidence), the wavelength dependence in $\Delta n_{\text {form }}$ should be tuned to be closely proportional to the wavelength across a wide spectral band.

\section{B. Rigorous Coupled Wave Analysis (RCWA)}

Common scalar theories of diffraction in gratings do not work in the subwavelength domain. In order to simulate the grating response and to calculate its form birefringence, we must consider the vectorial nature of light. For this purpose, we have performed numerical simulations using the RCWA. RCWA is an analysis method that is applicable to any multilayer grating profile $[12,13]$. The algorithm converts the grating-diffraction problem into a matrix problem, and solves the Maxwell equations from layer to layer. The solution corresponds to the reflection and transmission matrices in the two modes (TE/TM), describing the entire diffractive characteristics of the simulated structure: the diffraction efficiencies $\left(\eta_{\mathrm{TE}}^{(m)}\right)$ and $\left(\eta_{\mathrm{TM}}^{(m)}\right)$, and the phase shift $\left(\Delta \Phi_{\mathrm{TE}-\mathrm{TM}}\right)$.

\section{Choosing an Appropriate Material: Diamond}

For an HWP to keep its efficiency over a wide spectral band, the component must be optimized in order to minimize the phase shift error with respect to $\pi$ :

$$
\varepsilon(\lambda)=\Delta \Phi_{\mathrm{TE}-\mathrm{TM}}(\lambda)-\pi .
$$

Since the error is a function of the wavelength, we minimize the root mean squared (RMS) error $\varepsilon_{\text {rms }}$ 
over the entire spectral band. We have performed RCWA simulations to optimize the grating parameters for a refractive index ranging from 1.5 to 2.8, and for several specific mid-IR spectral bands, corresponding to the transmission windows of the Earth atmosphere: L $(3.5-4.1 \mu \mathrm{m}), \mathrm{M}(4.5-5.1 \mu \mathrm{m})$, and $\mathrm{N}(8-13 \mu \mathrm{m})$. Other solutions beyond $3 \mu \mathrm{m}$ are cumbersome and involve exotic materials. Considering an ideal rectangular profile, we optimized the parameters $F, h$, and $\Lambda$ (Fig. $\underline{1}$ ) using a multidimensional nonlinear minimization method (Nelder-Mead algorithm, also called downhill simplex method) from the MATLAB software. The period was constrained to be smaller than the SWG limit $\lambda / n$. With these optimized parameters, the mean phase shift error is very small $\left(\varepsilon_{\mathrm{rms}} \simeq 10^{-4} \mathrm{rad}\right)$ in the considered spectral bands. We also introduced another parameter, the aspect ratio $\rho$,

$$
\rho=\frac{h}{\min [F \Lambda ;(1-F) \Lambda]},
$$

which is the height-to-width ratio of either the walls or the grooves. This parameter must be made as small as possible since features with high aspect ratio are difficult to manufacture. In our simulations, the N-band was separated into two parts of comparable bandwidth $(\sim 20 \%)$, thereby avoiding the strong ozone absorption band around $10 \mu \mathrm{m}$. In Fig. $\underline{2}$, the optimal aspect ratio is plotted against refractive index. Minima in these plots indicate a suitable substrate refractive index for the grating. For the longest wavelengths ( $\mathrm{N}$-band mostly), the aspect ratio $\rho$ is much lower for high refractive indices $(>2.25)$, while for the shorter wavelengths, the filling factor $F$ varies strongly, which results in a very fluctuating aspect ratio due to its definition as a minimum of either the wall or void thickness. We conclude that using substrates with high refractive indices (e.g., diamond,

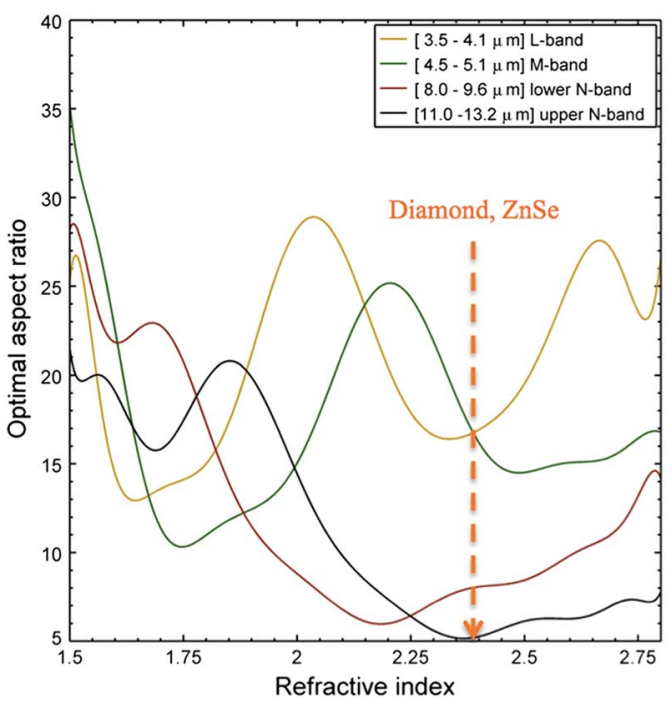

Fig. 2. (Color online) RCWA simulation: the aspect ratio of the gratings for a minimized mean phase shift error as a function of the refractive index.
ZnSe, Ge) can make the microfabrication of SWGs for achromatic HWPs much easier. Some exotic materials with lower refractive index exist, but they are not compatible with our applications because of many disadvantages (brittle, deform easily, etc.). Materials with very high refractive index such as silicon $(\sim 3.4)$ are not compatible either, because they necessarily require complex antireflective coatings on top of the SWG.

Among the materials commonly used in the $\mathrm{N}$ band, one good candidate is diamond, with a refractive index $\sim 2.38$ for the region of interest (from 3 to $13 \mu \mathrm{m}$ ). The use of diamond substrates leads to many advantages. First and foremost, it has a wide transmission window [14]. In addition, its mechanical properties are outstanding (low density $=3.52 \mathrm{~kg} / \mathrm{m}^{3}$; very high hardness $=10$ on the Mohs scale; very high elasticity). Also, its thermal (excellent conductor, inertia) and chemical (resistant to usual chemicals, acids, and most of the alkalies) properties make it an excellent candidate to be space qualified.

\section{Design of a Diamond Wave Plate}

The larger periods and lower aspect ratios of the $\mathrm{N}$-band HWP should make this grating easier to fabricate compared to bands of shorter wavelengths, as shown in Section 3. Here we will focus on a HWP for the long-wavelength part of the N-band (11-13.2 $\mu \mathrm{m})$ for a forthcoming astronomical application (VLT/ VISIR) [15]. The design of the grating was conducted in synergy with the manufacturing [16]. In particular, the slope of the sidewalls (see Fig. 3) must be taken into account and the aspect ratios must be kept within the range of what can be etched in the material.

The etch process used induces a slope $\alpha \simeq 2.7^{\circ}$ (Fig. 3). During the fabrication process, small errors in line width, slope, and grating depth occur. In particular, the depth $h$ and the slope $\alpha$ are difficult to measure precisely. A design that performs well even under small changes in these parameters was therefore sought. We have computed two-dimensional (2D) maps of the RMS error $\left(\varepsilon_{\text {rms }}\right)$ as a function of the filling factor $(F)$ and of the depth $(h)$, for several values of $\alpha$ ranging from 2.6 to $2.8^{\circ}$. We also calculated the mean and standard deviation of all these maps (see Fig. 4).

To obtain a good compromise between the mean value and the standard deviation, the optimum values are $h=13.7 \mu \mathrm{m}$ and $F=0.4$, which correspond to a line width (on the top) $F \Lambda=1.84 \mu \mathrm{m}$. In the first

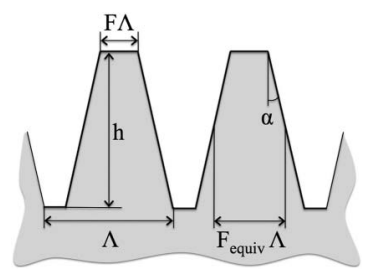

Fig. 3. Schematic diagram of a trapezoidal grating. The grating walls have a slope $\alpha$ and an average width $F_{\text {equiv }} \Lambda$. 

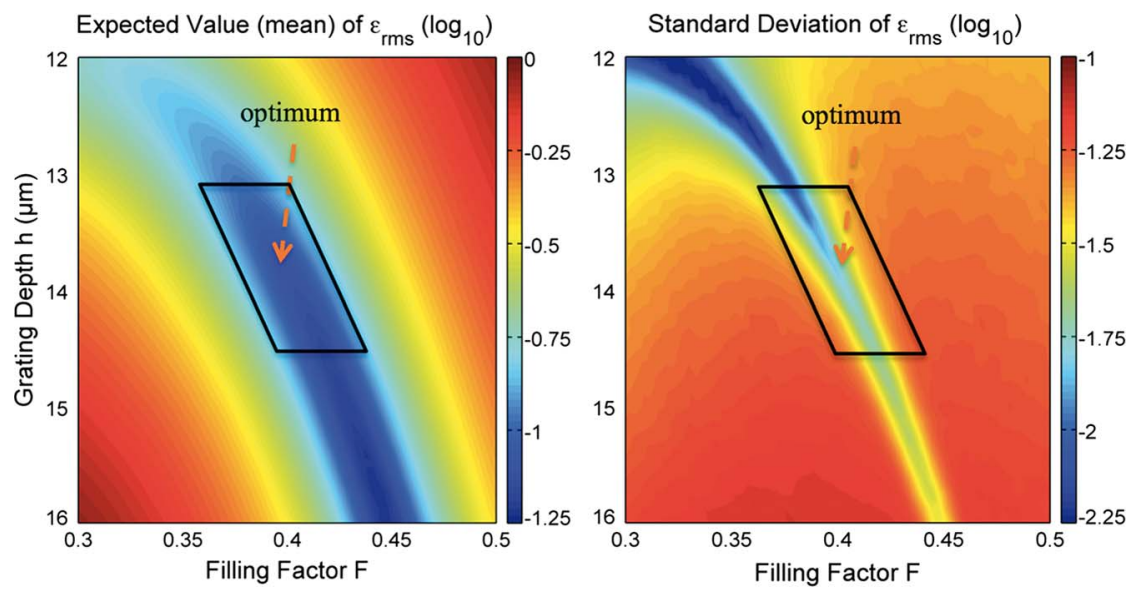

Fig. 4. (Color online) RCWA multiparametric simulation: mean (left) and standard deviation (right) of the RMS phase shift error (logarithmic scale) over the upper N-band, with $\alpha$ ranging from 2.6 to $2.8^{\circ}$. The period is set to $\Lambda=4.6 \mu \mathrm{m}$ (SWG limit).

approximation, one can tell that the relation between the optimum line width and depth is quasi linear, with a regression coefficient approximately equal to 10 . This is very useful for the manufacturing process, to compensate line width errors with the etching depth. For instance, if the line width is smaller than the optimum (e.g., $-50 \mathrm{~nm})$, one can etch a shallower grating $(-500 \mathrm{~nm})$.

\section{Manufacturing and Performance Analysis}

\section{A. Manufacturing}

The pattern was first written in photoresist on a silicon wafer by direct laser writing. This pattern was then transferred to the diamond substrate using a nanoimprint lithography process and finally dry etched in the diamond via several masking layers in an inductively coupled plasma reactor. The details of this process will be published elsewhere. The dense oxygen/argon plasma used is very stable, but the etch rate varies with depth and width of the grooves.

As mentioned before, precise measurements of the depth and profile of the subwavelength structure are difficult. The grooves are too narrow for using atomic force microscopy or white light interferometry. The best measurements were obtained by cracking the sample and observing the cross section with a scanning electron microscope (SEM). As can be seen in Fig. 5 the sidewalls have a slight angle of $2.6-2.8^{\circ}$ from the vertical. Ions deflected in glancing impacts with the sidewalls cause trenching at the bottom of the wall. In a narrow groove such as this, trenching gives rise to the triangular ridge along the center of the groove.

\section{B. Expected Performances}

Cracking the sample is naturally not possible for the final HWPs. For these an estimate of the depth was acquired by comparing several SEM images taken at different angles. The distance between two recognizable features, one at the top and one at the bottom of a groove, was measured in micrographs recorded at tilt angles between 5 and $26^{\circ}$. From the variation in this distance with angle, the depth could be calculated. The depth measured by this method on cracked samples was in good agreement with cross section images (within 2\%). Our prototypes have met the specifications: line width $\simeq 1.8 \mu \mathrm{m}$ (sidewall angle $\simeq 2.7^{\circ}$ ), period $4.6 \mu \mathrm{m}$, and depth $\simeq 13.7 \mu \mathrm{m}$. As shown in Fig. 6 , the retardance of the manufactured HWP is nearly ideal in the center of the spectral band. The mean and standard deviation of the phase shift over the whole upper N-band $(11-13.2 \mu \mathrm{m})$ equal $\sim 3.143 \pm 0.061 \mathrm{rad}\left(180.08 \pm 3.51^{\circ}\right)$.

\section{Antireflective Grating (ARG)}

Incoherent reflections with different phase shift may interfere with the main beam and degrade performance of the component. The natural reflection of the diamond at N-band is $\sim 17 \%$ for one interface. Although the achromatic HWP was not designed with reducing reflections in mind, it still serves this purpose to some degree. As shown in Fig. 7, the theoretical transmittance is quite good between 11.5 and $13 \mu \mathrm{m}$ because the diffraction grating actually acts as an antireflective layer at this wavelength.

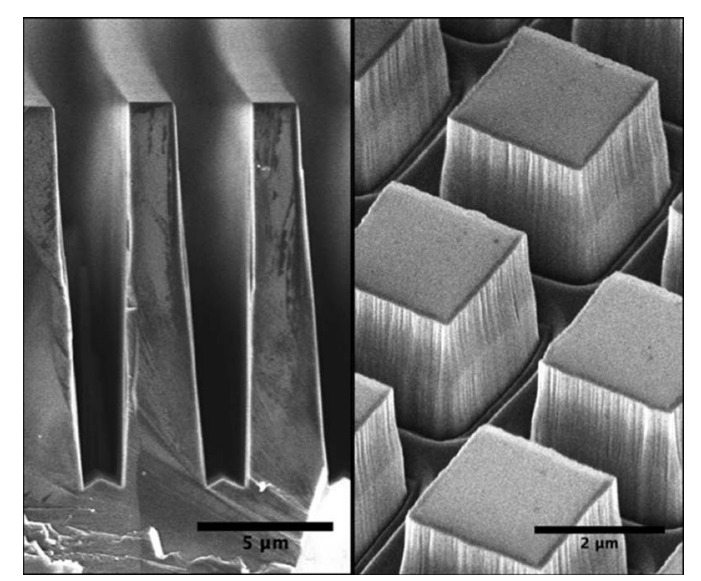

Fig. 5. SEM-micrographs of a diamond achromatic HWP. Left: cross sectional view of the grooves. Right: antireflective structure on the backside. 


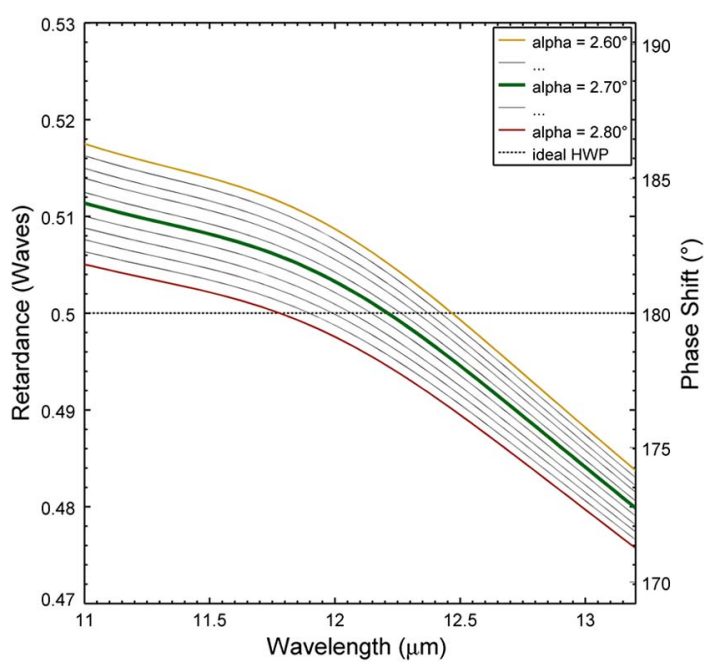

Fig. 6. (Color online) Retardance for a diamond HWP with optimal specifications at $\alpha=2.6-2.8^{\circ}$ (slope of the walls). Bandwidth: $11-13.2 \mu \mathrm{m}$.

In addition, an ARG was designed to reduce surface reflections using a diffraction grating analysis program (GSOLVER, version 4.20c., Grating Solver Development Co., USA). The program uses algorithms based on RCWA to calculate values of the zero-order transmission. A 2D SWG formed by binary square $(2.6 \times 2.6 \times 2 \mu \mathrm{m})$ shaped structures with a $4 \mu \mathrm{m}$ period was etched on the backside of the HWP to reduce the reflection from $17 \%$ to less than $0.5 \%$ in the wavelength region of interest (see Fig. 8). Etching the ARG was much less demanding than the HWP structure, but there is still trenching and the sidewalls are not perfectly vertical [11] (Fig. 5). Since there was some deviation from the calculated structure, the etch time was optimized by testing the performance. The transmittance of

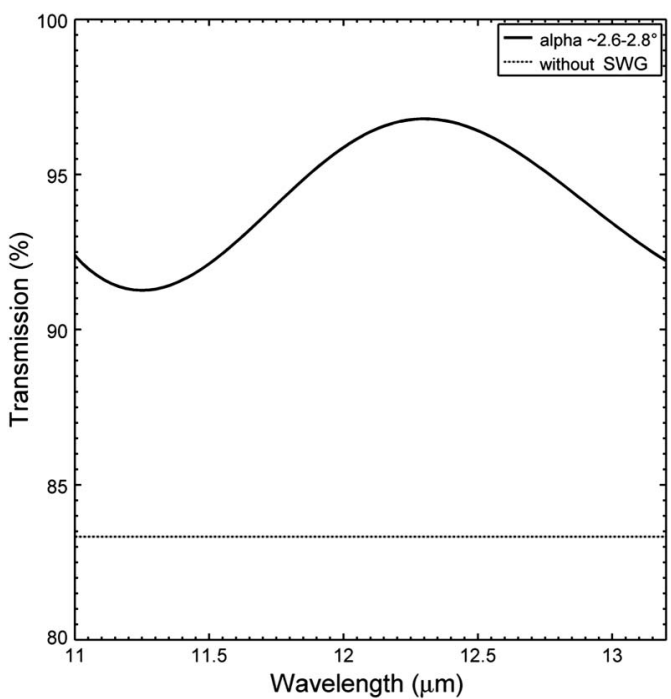

Fig. 7. Transmission spectrum of a SWG etched on diamond with HWP optimal specifications, with sidewall angle $\left(2.6-2.8^{\circ}\right)$. The dotted line shows the natural transmission of the diamond at N-band, without the SWG $(\sim 83 \%)$.

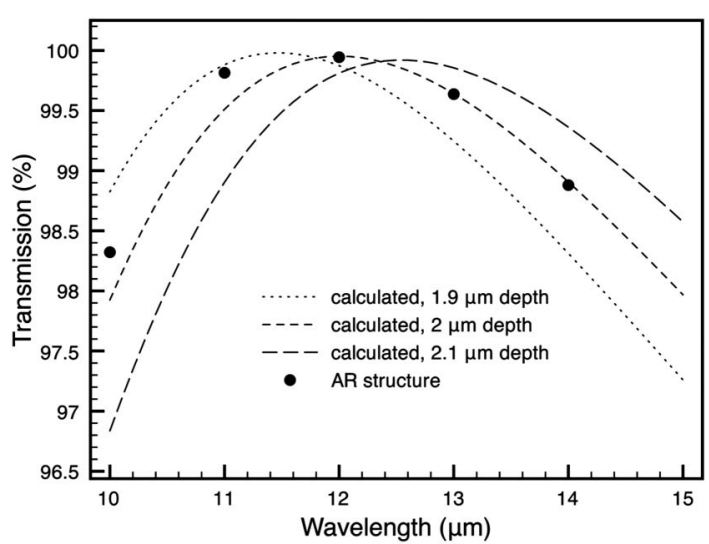

Fig. 8. Transmission spectrum of one diamond interface with ARG measured with a spectrophotometer. The calculated transmission values for three different depths are also shown.

diamond substrates with a single sided ARG was measured in a Perkin Elmer 983 infrared spectrophotometer. After removing the effects of interference within the sample and comparing with an unetched sample, the transmittance of a single AR interface has been determined (Fig. 8). As can be seen, the bandwidth of the manufactured structure was slightly larger than the calculated one. This may be due to the somewhat tilted sidewalls. The total transmittance of the finished HWP components was between $89 \%$ and $95 \%$ over the band.

\section{Conclusion and Directions for Future Research}

Diamond is a good material for manufacturing achromatic HWPs for mid-IR wavelengths. By optimizing the gratings with manufacturing limitations in mind, components dedicated to the upper N-band $(\sim 12 \mu \mathrm{m})$ could be achieved including an antireflective solution on their backside. Diamond HWPs are likely to be used for many applications, particularly for optical vortices in astrophysics. The diamond HWP shown in this paper has been developed to enable the manufacturing of an N-band annular groove phase mask (AGPM), which will be installed in 2012 on the VISIR instrument at the VLT, and is also an excellent candidate for METIS at the future European Extremely Large Telescope. This specific application will be the subject of a forthcoming paper. Moreover, a new process using e-beam lithography is now being explored to reach smaller grating periods, and thereby shorter operating wavelengths. These AGPMs are being evaluated for implementation on high-contrast imaging instruments such as NACO (L-band $\sim 3.8 \mu \mathrm{m}$ ) and SPHERE (K-band $\sim 2.2 \mu \mathrm{m}$ ) at the Very Large Telescope in Chile.

The first author is grateful to the financial support of the Belgian Fonds de la Recherche Scientifique (FRIA) and Fonds de solidarité ULg. We also gratefully acknowledge financial support from the Swedish Diamond Center (financed by Uppsala University), and the Communauté française de 
Belgique-Actions de recherche concertées-Académie universitaire Wallonie-Europe.

\section{References}

1. R. M. A. Azzam and C. L. Spinu, "Achromatic angleinsensitive infrared quarter-wave retarder based on total internal reflection at the $\mathrm{Si}_{-} \mathrm{SiO}_{2}$ interface," J. Opt. Soc. Am. A 21, 2019-2022 (2004).

2. J.-B. Masson and G. Gallot, "Terahertz achromatic quarterwave plate," Opt. Lett. 31, 265-267 (2006).

3. S. Pancharatnam, "Achromatic combinations of birefringent plates-Part II. An achromatic quarter wave plate," Proc. Indian Acad. Sci. A 42, 24-31 (1955).

4. J. Schirmer and T. Schmidt-Kaler, "Liquid crystal phase retarder with broad spectral range," Opt. Commun. 176, 313-317 (2000).

5. D. Mawet, E. Serabyn, K. Liewer, Ch. Hanot, S. McEldowney, D. Shemo, and N. O'Brien, "Optical vectorial vortex coronagraphs using liquid crystal polymers: theory, manufacturing and laboratory demonstration," Opt. Express 17, 1902-1918 (2009).

6. D. R. Solli, C. F. McCormick, and J. M. Hickmann, "Polarizationdependent reflective dispersion relations of photonic crystals for waveplate mirror construction," J. Lightwave Technol. 24, 3864-3867 (2006).

7. N. Murakami, J. Nishikawa, K. Yokochi, M. Tamura, N. Baba, and L. Abe, "Achromatic eight-octant phase-mask coronagraph using photonic crystal," Astrophys. J. 714, 772-777 (2010).

8. D. Mawet, E. Serabyn, K. Liewer, R. Burruss, J. Hickey, and D. Shemo, "The vector vortex coronagraph: laboratory results and first light at Palomar Observatory," Astrophys. J. 709, 53-57 (2010).
9. H. Kikuta, Y. Ohira, and K. Iwata, "Achromatic quarter-wave plates using the dispersion of form birefringence," Appl. Opt. 36, 1566-1572 (1997).

10. G. Nordin and P. Deguzman, "Broadband form birefringent quarter-wave plate for the mid-infrared wavelength region," Opt. Express 5, 163-168 (1999).

11. M. Karlsson and F. Nikolajeff, "Diamond micro-optics: microlenses and antireflection structured surfaces for the infrared spectral region," Opt. Express 11, 502-507 (2003).

12. M. G. Moharam and T. K. Gaylord, "Rigorous coupled-wave analysis of planar-grating diffraction," J. Opt. Soc. Am. 71, 811-818 (1981).

13. L. Li, "Multilayer modal method for diffraction gratings of arbitrary profile, depth, and permittivity," J. Opt. Soc. Am. 10, 2581-2591 (1993).

14. F. P. Bundy, "Melting point of graphite at high pressure: heat of fusion," Science 137, 1055-1057 (1962).

15. P. O. Lagage, J. W. Pel, M. Authier, J. Belorgey, A. Claret, C. Doucet, D. Dubreuil, G. Durand, E. Elswijk, P. Girardot, H. U. Käufl, G. Kroes, M. Lortholary, Y. Lussignol, M. Marchesi, E. Pantin, R. Peletier, J.-F. Pirard, J. Pragt, Y. Rio, T. Schoenmaker, R. Siebenmorgen, A. Silber, A. Smette, M. Sterzik, and C. Veyssiere, "Successful commissioning of VISIR: the mid-infrared VLT Instrument," The Messenger 117, 12-16 (2004).

16. C. Delacroix, P. Forsberg, M. Karlsson, D. Mawet, C. Lenaerts, S. Habraken, C. Hanot, J. Surdej, A. Boccaletti, and J. Baudrand, "Annular groove phase mask coronagraph in diamond for mid-IR wavelengths: manufacturing assessment and performance analysis,” Proc. SPIE 7731, 77314W (2010). 